\begin{tabular}{|c|l|}
\hline Title & Effect of feeding regimens on poly hydroxybutyrate production from food wastes by Cupriavidus necator \\
\hline Author(s) & Hafuka, A kira; Sakai da, Kenji; Satoh, Hisashi; Takahashi, Masahiro; W atanabe, Y oshimasa; Okabe, Satoshi \\
\hline Citation & $\begin{array}{l}\text { BIORESOURCE TECHNOLOGY, 102(3), 3551-3553 } \\
\text { https://doi.org/L0.1016j.biortech.2010.09.018 }\end{array}$ \\
\hline Issue Date & 2011-02 \\
\hline Doc URL & http://hdl.handle.net/2115/44977 \\
\hline Type & article (author version) \\
\hline File Information & sato.pdf \\
\hline
\end{tabular}

Instructions for use 


\title{
Effect of feeding regimens on polyhydroxybutyrate production from food wastes by Cupriavidus necator
}

\author{
Akira Hafuka, Kenji Sakaida, Hisashi Satoh*, Masahiro Takahashi, Yoshimasa \\ Watanabe, and Satoshi Okabe
}

Division of Environmental Engineering, Faculty of Engineering, Hokkaido University, North-13, West-8, Sapporo 060-8628, Japan.

*Corresponding author.

Mailing address:

Hisashi Satoh

Division of Environmental Engineering, Faculty of Engineering, Hokkaido University, North-13, West-8, Sapporo 060-8628, Japan.

Tel: +81-(0)11-706-6277

Fax: +81-(0)11-706-6277

E-mail: qsatoh@eng.hokudai.ac.jp

List of abbreviations: PHA, polyhydroxyalkanoate; PHB, polyhydroxybutyrate; DO, dissolved oxygen; DCW, dry cell weight; COD, chemical oxygen demand; VFA, volatile fatty acid; D-COD, dissolved chemical oxygen demand; GC, gas chromatography; PHB-PHV, poly(3-hydroxybutyrate-co-3-hydroxyvalerate) 


\begin{abstract}
We investigated the effects of different feeding regimens (1-pulse, stepwise, and continuous) of fermented food-waste liquid on polyhydroxybutyrate (PHB) production. The fermentation liquid was filtered with a membrane filter (pore size, $0.45 \mu \mathrm{m}$ ) to remove anaerobic microorganisms and solids and used as a carbon source for Cupriavidus necator. One-pulse feeding yielded the highest cell concentration of $C$. necator. However, the PHB concentration was higher in the stepwise- and continuous-feeding regimens. Therefore, the continuous-feeding regimen was used for continuous PHB production. PHB could be produced over 259 h ( 8 draw-fill cycles) with a maximal PHB content of $87 \%$, but the PHB concentration and content decreased with an increase in the operation time.
\end{abstract}

Keywords: polyhydroxybutyrate; food wastes; filtration; feeding regimens; continuous PHB production 


\section{Introduction}

The use of petroleum-based synthetic plastics has led to problems in the management of solid waste. Therefore, bio-based and biodegradable polymers such as polyhydroxyalkanoates (PHAs) have attracted considerable attention as alternatives to the petroleum-based plastics (Castilho et al., 2009). In almost all established industrial processes for PHA production, the substrates used are sugar-based compounds, which have a high market price (Serafim et al., 2008). Therefore, more cost-effective technologies are required. In recent years, there has been a growing interest in the production of PHAs by using low-value substrates, such as wastes and wastewaters (Koller et al., 2008). These substrates are generally utilized for PHA production in mixed microbial cultures (Rodgers and Wu, 2010). This technique is more energy-efficient than that involving pure cultures (Kek et al., 2008; Lee et al., 2008). However, the PHA content in the mixed cultures was not high $(<65 \%)$ (Serafim et al., 2008). In contrast, PHA production by pure cultures yields a very high PHA content (ca. 80\%) (Li et al., 2007; Lee, 1996). Hence, these 2 different processes should be coupled in a more cost-effective method.

Du and Yu (2002) proposed a technique wherein membrane separation technology was used to transfer organic acids obtained from a food-waste fermentation reactor to a PHA production reactor with a pure culture without washout of anaerobic microorganisms and solids. However, the pore size of the membranes used was too small for the removal of anaerobic microorganisms and solids, resulting in high consumption of operational energy. Moreover, in the majority of the previous studies, PHAs were produced in only 1 
batch (Kek et al., 2008; Du and Yu, 2002). From an economic point of view, it would be beneficial to repeatedly use the pure culture as a seed culture for PHA production. The aim of this study was, therefore, to produce polyhydroxybutyrate (PHB), which is the most widespread and the best-characterized PHA, by using filtered fermented food wastes as carbon sources with a pure culture of Cupriavidus necator H16 (formerly Ralstonia eutropha) as the PHB-producing species. We investigated the effects of different regimens (1-pulse, stepwise, and continuous feeding regimens) for feeding the fermentation liquid on the efficiency of PHB production and achieved continuous $C$. necator-mediated production of PHBs from the fermented food waste liquid.

\section{Materials and methods}

Food wastes were collected from a canteen in Hokkaido University. Fermentation of the food waste slurry was performed at ambient temperature in a 2-L reactor with a working volume of 1.5 L. First, 0.5 L of the inoculum was mixed with $1.0 \mathrm{~L}$ of the fresh food waste slurry, and then the reactor was continuously operated under the draw-fill mode: 1.0 L of the slurry was withdrawn and replaced with $1.0 \mathrm{~L}$ of fresh food waste slurry once a week (see Figure 1A). Thus, the fermentation liquid for PHB production was obtained once a week. The fermentation liquid was filtered with a membrane filter with a pore size of $0.45 \mu \mathrm{m}$.

C. necator H16 (ATCC 17699) was used for PHB production. The strain was cultivated in ATCC medium 3 and cells were harvested by centrifugation. The cell pellet was then resuspended in $200 \mathrm{~mL}$ of the medium and subsequently used as the seed culture for PHB 
production. PHB was produced in fed-batch cultures of $C$. necator in 1.5 -L air-bubbling cylindrical reactors. The seed culture was added to 3 PHB production reactors. After diluting the seed culture with $500 \mathrm{~mL}$ of tap water, $150 \mathrm{~mL}$ of the filtered fermentation liquid was added to reactors $\mathrm{A} 1, \mathrm{~A} 2$, and $\mathrm{A} 3$ by using different feeding regimens. In reactor $\mathrm{A} 1$, the entire volume of the fermentation liquid was fed to the reactor as a single unit, i.e., the 1-pulse regimen (Run 1). In reactor A2, the fermentation liquid was fed in a stepwise manner once a day with 7 pulses (21 mL in each pulse) (Run 2). In reactor A3, the fermentation liquid was continuously fed to the reactor by using a peristaltic pump at a rate of $150 \mathrm{~mL} /$ week (21 mL/day) (Run 3). The PHB production reactors were operated at $20 \pm 1{ }^{\circ} \mathrm{C}$ with aeration of $0.3 \mathrm{vvm}$ and the $\mathrm{pH}$ was adjusted to 7.5 . Furthermore, continuous PHB production with $C$. necator was performed by using the protocol employed in Run 3.

Measurements of chemical oxygen demand (COD), volatile fatty acid (VFA) content and $\mathrm{pH}$ were performed as described in our previous publication (Satoh et al., 2007). The dissolved fraction of COD, which passed through a membrane filter with a pore size of $0.45 \mu \mathrm{m}$, was termed D-COD. The total VFA concentration (g-C/L) was calculated as the sum of the carbon content of the measured amounts of formic, acetic, propionic, lactic, $n$-butyric, and isobutyric acid. The dry cell weight (DCW) and PHB concentration and content were determined by using the method described by Braunegg et al. (1978). PHB concentration in samples was determined using a GC system (GC-14B; Shimadzu Co., Kyoto, Japan) equipped with a flame ionization detector and Inert CAP 5 column (30 m length $\times 0.25 \mathrm{~mm}$ internal diameter $\times 0.4 \mu \mathrm{m}$ film thickness) (GL Science, Tokyo, Japan). The PHB content in the DCW (w/w) was defined as the percentage of PHB in the DCW. 
The yields of DCW and PHB from VFAs were calculated as the ratios of mole carbon in DCW and PHB to mole carbon in VFAs, respectively. The chemical formulas of DCW and PHB were assumed to be $\mathrm{C}_{5} \mathrm{H}_{7} \mathrm{NO}_{2}$ and $\mathrm{O}-\mathrm{CH}\left(\mathrm{CH}_{3}\right)-\mathrm{CH}_{2}-\mathrm{CO}$, respectively.

\section{Results and discussion}

D-COD and VFAs increased with an increase in the food waste loading (Figure 1). In each cycle, these values reached a quasi-steady-state within 7 days of each draw-fill cycle. Acetic acid accounted for ca. $50 \%$ of the total VFA content at $500 \mathrm{~h}$. Thereafter, lactic acid was the largest component (ca. 80\% (in mg-C/L)) in the first $720 \mathrm{~h}$. After $\mathrm{pH}$ adjustment (after $720 \mathrm{~h}$ ), lactic acid still remained the most dominant component. However, acetic acid and propionic acid concentrations increased after 1,120 h, probably because of a change in the anaerobic microbial consortia. The predominance of lactic acid at $\mathrm{pH}$ lower than 4 and the relative increase in acetic acid and propionic acid concentrations after the adjustment of $\mathrm{pH}$ to around 6 were consistently shown in triplicate experiments (data not shown) and in previous studies (Du and Yu, 2002). PHB seems to be effectively produced from acetic acid (Serafim et al., 2008). Moreover, propionic acid production is also beneficial because it leads to the synthesis of a poly(3-hydroxybutyrate-co-3-hydroxyvalerate) (PHB-PHV) copolymer (Du and Yu, 2002), which is more ductile and tougher than the PHB homopolymer (Lee et al., 2008). These findings indicate that acetic and propionic acids production in the fermentation process is beneficial for effective PHB and PHB-PHV copolymer production. The changes in DCW, VFA concentration, and PHB concentration and content in the 
PHB production reactors fed with the filtered fermentation liquid were investigated with different feeding regimens. Feeding in the 1-pulse regimen (Run 1) resulted in a greater extent of $C$. necator growth (10,000 mg-DCW/L), probably because of higher initial VFA levels. This value was comparable with those reported in previous studies ( Du and Yu, 2002; Lee et al., 2008). In contrast, it was lower than those reported in several other studies (Li et al., 2007), probably because of lower organic loading rate and/or the use of a wild-type strain instead of recombinant strains. In contrast, the maximal PHB concentrations and contents in Runs $2(250 \mathrm{mg} / \mathrm{L}$ and $25 \%$ at $44 \mathrm{~h})$ and $3(200 \mathrm{mg} / \mathrm{L}$ and $24 \%$ at $40 \mathrm{~h})$ were higher than that in Run $1(65 \mathrm{mg} / \mathrm{L}$ and $3 \%$ at $46 \mathrm{~h})$. It was obvious that the application of the stepwise and continuous feeding regimens yielded PHB concentration and content higher than those achieved by using the 1-pulse feeding regimen. The higher PHB content is beneficial because of the consequent less production of wastes (i.e., residual biomass and $\mathrm{CO}_{2}$ ) in the PHB production process. It is known that net PHB accumulation takes place effectively under nutrient-limited conditions [e.g., high carbon-to-nitrogen ratio $(\mathrm{C} / \mathrm{N})]$ (Castilho et al., 2009). Indeed, the $\mathrm{NH}_{4}{ }^{+}-\mathrm{N}$ concentration was $123 \mathrm{mg}-\mathrm{N} / \mathrm{L}$ at $0 \mathrm{~h}$ in Run 1 while it was not detected throughout the operation in Run 3. Thus, the $\mathrm{C} / \mathrm{N}$ ratios during the operation might be in the order of Run $3>$ Run $2>$ Run 1. 

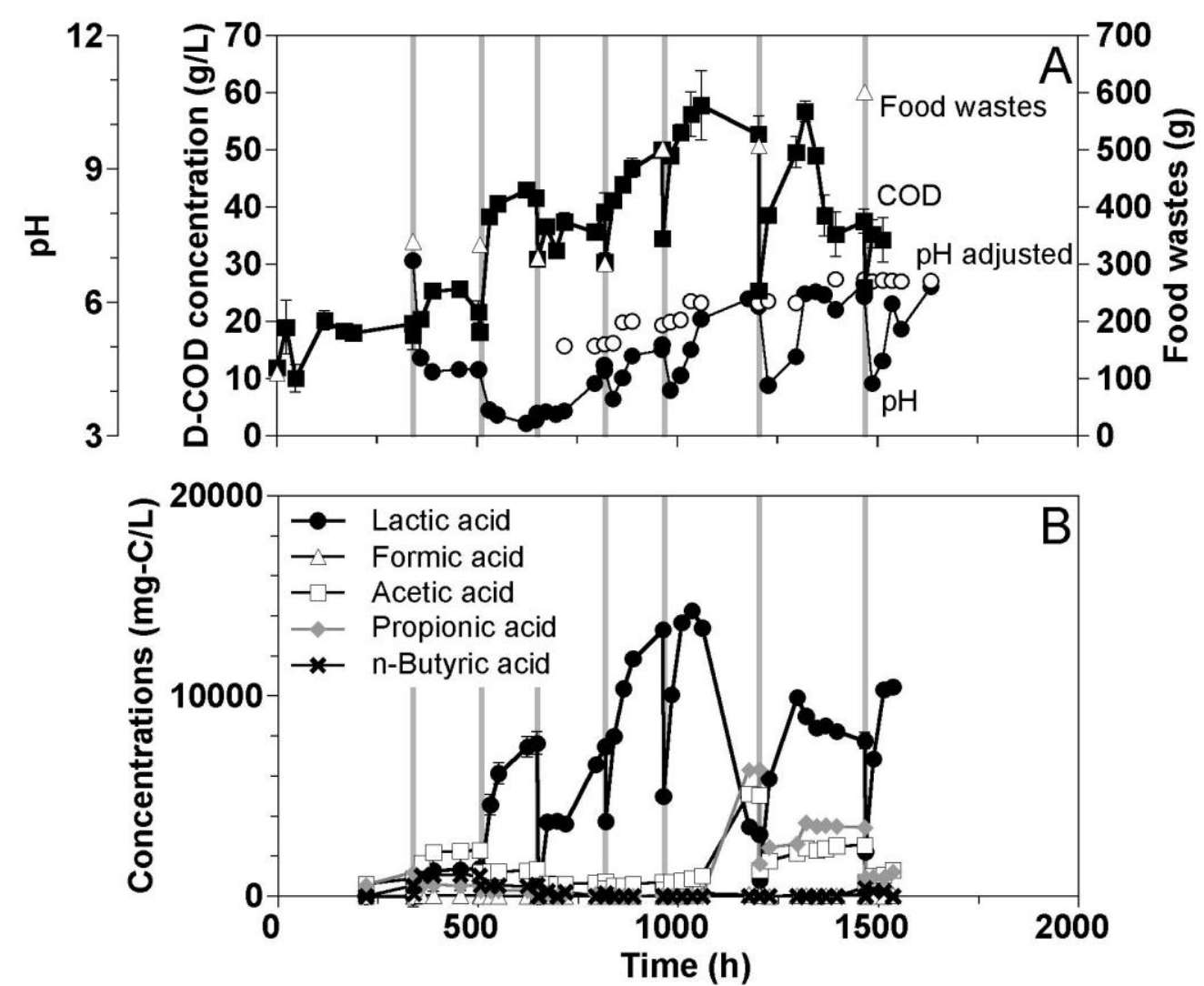

Figure 1. The typical time course of changes in the addition of food wastes, dissolved chemical oxygen demand (D-COD), $\mathrm{pH}$ before and after adjustment (A), and volatile fatty acid (VFA) concentrations (B) during continuous fermentation of food wastes. The timing of withdrawal of the slurry and addition of fresh food waste are indicated by gray lines.

C. necator-mediated PHB production from the filtered fermentation liquid was performed. The DCW and PHB concentrations were the highest at $43 \mathrm{~h}$ (Figure 2A). The DCW production rates were relatively high in the initial operation and gradually decreased (Figure 2B). The PHB production rates were also high in the initial operation and decreased after $188 \mathrm{~h}$, but PHB production continued at a rate of $5 \mathrm{mg} \cdot \mathrm{L}^{-1} \cdot \mathrm{h}^{-1}$ even at $259 \mathrm{~h}$ (at the 8th cycle). The PHB content $(<87 \%)$ was higher than that produced from the 
filtered fermentation liquid ( $\mathrm{Du}$ and $\mathrm{Yu}, 2002$ ) and comparable to that obtained from pure feedstock (Li et al., 2007; Lee, 1996).
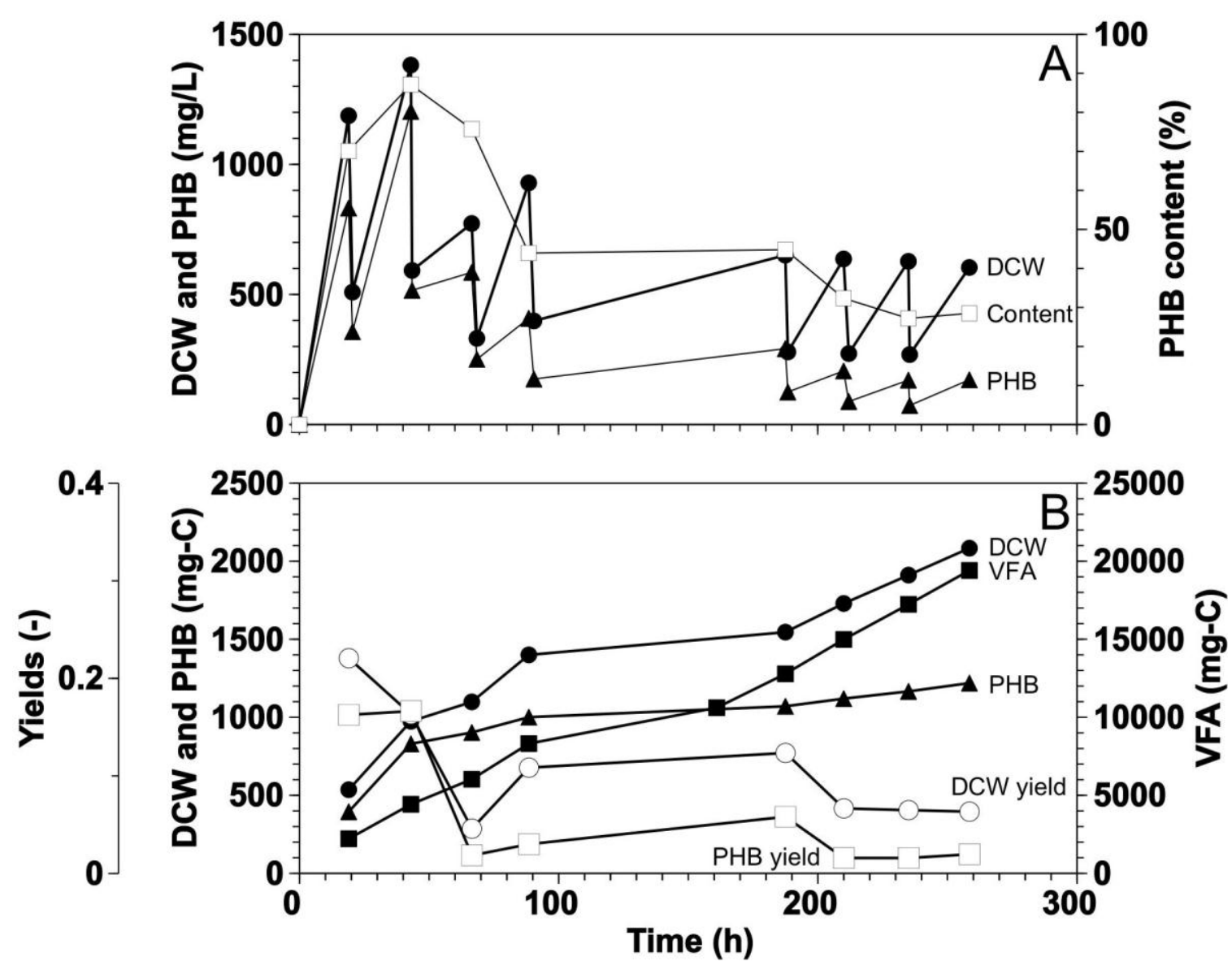

Figure 2. Variations in dry cell weight (DCW) and polyhydroxybutyrate (PHB) concentrations and PHB content in the PHB production reactor (A). PHB production from the filtered fermentation liquid by $C$. necator was performed in duplicate (B). The cumulative amounts of DCW produced, PHB produced, and total VFAs consumed, and the DCW and PHB yields in the PHB production reactor were calculated from the results in the duplicate operation (C). 


\section{Conclusions}

This study showed that PHB could be continuously produced from fermented food wastes filtered through a $0.45-\mu \mathrm{m}$-pore-size membrane filter without using any other expensive pretreatment measures and by repeatedly using a pure microbial culture. The problem with contamination of the PHB reactor with anaerobes were overcome by that the anaerobes were removed by using a $0.45-\mu \mathrm{m}$-pore-size membrane filter. Among the 3 different feeding regimens tested, the continuous feeding regimen seemed to be the most suitable for effective PHB production. Further studies are necessary to develop methods to maintain the high rate of $\mathrm{PHB}$ production.

\section{Acknowledgements}

We thank Dr. Mamoru Oshiki for valuable discussions during the preparation of the manuscript. This work was partially supported by the funding for Basic and Applied Researches on Construction Technologies from the Ministry of Land, Infrastructure, Transport, and Tourism of Japan.

\section{References}

1. Braunegg, G., Sonnleitner B., Lafferty R.M., 1978. A rapid gas chromatographic method for the determination of poly- $\beta$-hydroxybutyric acid in microbial biomass. Eur. J. Appl. Microbiol. Biotechnol. 6, 29-37.

2. Castilho, L.R., Mitchel, D.A., Freire, D.M.G., 2009. Production of 
polyhydroxyalkanoates (PHAs) from waste materials and by-products by submerged and solid-state fermentation. Bioresour. Technol. 100, 5996-6009.

3. Du, G., Yu, J., 2002. Green technology for conversion of food scraps to biodegradable thermoplastic polyhydroxyalkanoates. Environ. Sci. Technol. 36, $5511-5516$

4. Kek, Y.K., Lee, W.H., Sudesh, K., 2008. Efficient bioconversion of palm acid oil and palm kernel acid oil to poly(3-hydroxybutyrate) by Cupriavidus necator. Can. J. Chem. Rev. Can. Chim. 86, 533-539.

5. Koller, M., Bona, R., Chiellini, E., Fernandes, E.G., Horvat, P., Kutschera, C., Hesse, P., Braunegg, G., 2008. Polyhydroxyalkanoate production from whey by Pseudomonas hydrogenovora. Bioresour. Technol. 99, 4854-4863.

6. Lee, S.Y., 1996. Plastic bacteria? Progress and prospects for polyhydroxyalkanoate production in bacteria. Trends Biotechnol. 14, 431-438.

7. Lee, W.H., Loo, C.Y., Nomura, C.T., Sudesh, K., 2008. Biosynthesis of polyhydroxyalkanoate copolymers from mixtures of plant oils and 3-hydroxyvalerate precursors. Bioresour. Technol. 99, 6844-6851.

8. Li, R., Zhang, H.X., Qi, Q.S., 2007. The production of polyhydroxyalkanoates in recombinant Escherichia coli. Bioresour. Technol. 98, 2313-2320.

9. Rodgers, M., Wu, G.X., 2010. Production of polyhydroxybutyrate by activated sludge performing enhanced biological phosphorus removal. Bioresour. Technol. 101, 1049-1053.

10. Satoh, H., Miura, Y., Tsushima, I., Okabe, S., 2007. Layered structure of bacterial and archaeal communities and their in situ activities in anaerobic granules. Appl. 
Environ. Microbiol. 73, 7300-7307.

11. Serafim, L.S., Lemos, P.C., Albuquerque, M.G.E., Reis, M.A.M., 2008.

Strategies for PHA production by mixed cultures and renewable waste materials. Appl. Microbiol. Biotechnol. 81, 615-628. 